\title{
Classification of Different Cancer Types by Deep Convolutional Neural Networks
}

\author{
H.S. Nogay
}

\begin{abstract}
In this study, ten different types of cancer were classified with deep convolutional neural networks (DCNN). A total of 10,000 MRI (Magnetic Resonance Imaging) data were used for ten cancer patients, including 1000 MRI data for each cancer type. Although the images were reduced to $28 \times 28$ pixels, the DCNN model performed classification with an accuracy rate of 0.98 after 27 seconds and 15 epochs of training. The error rate in the last epoch in the study is also very close to zero. A highly successful classification has been achieved with the proposed DCNN model.
\end{abstract}

Index Terms-Deep Convolutional Neural Network, Cancer Types, Classification, Accuracy

\section{INTRODUCTION}

$\mathrm{C}$ ONVOLITIONAL neural networks, which are recently enhanced and improved artificial neural networks, are one of the deep learning approaches used in many applications such as image recognition, classification and pattern recognition [1]. Nowadays, deep convolutional neural networks (DCNN) are used especially in the diagnosis and classification of fatal diseases such as cancer [1 - 4]. Cancer patients often learn by chance that they are cancerous. Because when they go to the doctor, the results of the examinations done to them are usually for the evaluation of other possibilities. People generally do not do any analysis unless they are sick or doctors need it. Early diagnosis of cancer is therefore very difficult. Which cancer type the cancer patients have is revealed by the doctor examining the patient's MR images or clinical data. However, since the metastases are so insidious, they are noticed very late. Knowing what is cancerous in a very practical and fast manner with MR images on hand is then very useful for early diagnosis before metastasis. Classification of information on different types of cancer in MR images may contribute to the understanding of metastatic conditions in the future together with their reasons for the examination and prophylaxis. In this study, it was aimed to classify cancer types with deep learning approach using ten thousand data of ten types of cancer patients. One thousand data of each cancer type was used. In the study, image patches recorded in jpg format are used. As a result, a fairly satisfactory classification rate has been achieved.

H. S NOGAY, is with Mustafa Cikrikcioglu Vovational College, Erciyes University, Kayseri, Turkey, (e-mail: nogay@erciyes.edu.tr)

Manuscript received September 13, 2017; accepted January 08, 2018. DOI: $10.17694 /$ bajece. 410250

\section{MATERIALS}

The data used in this study were obtained from The Cancer Imaging Archive (TCIA) website [5, 6]. In the study, 10.000 pieces of jpg format image data were used for 10 cancer types. In order to create the data set, 1000 image data of each cancer patient were collected and recorded separately. Figure 1 shows the data set and labels used in the study. In the data set shown in Figure 1, abbreviations such as the following are used as labels. In addition, each cancer type is numbered from zero to nine for classification.

BRN : Brain cancer $(0)$

BRST : Breast cancer (1)

COLN: Colon cancer (2)

HN: Head and neck cancer (3)

L : Lung cancer (4)

PROS: Prostate cancer (5)

PAC: Pancreas cancer (6)

RENL: Renal cancer (7)

SRKO: Sarkomas (8)

TROID: Thyroid cancer (9)

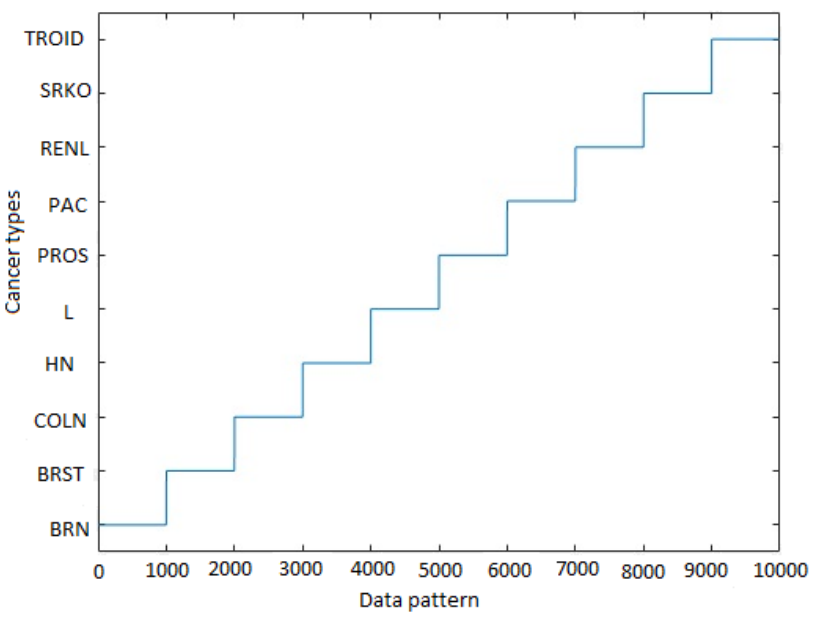

Fig. 1. Data set configuration

Each image patch in the data set is actually $216 \times 256$ pixels as shown in figure 2 in the sample brain MR image. All image patches have been reduced to a $28 \times 28$ size so that the operation can be faster and the data set is not overly saturated. 
A sample dataset of $28 \times 28$ image patches consisting of 20 random data samples is shown in Fig. 3 .

All images used in the study are only black and white, that is two-layer images. The aim is to determine which cancer type belongs to the MRI data, so no 3 or more layered images are needed. Rapid training of the model depends on whether the operating system to be used is GPU-supported, the number of data in the data set, the pixel of the data and whether it is layered. When all of these factors are considered, it can be seen that preferences made for the DCNN model are only considered to achieve rapid results.

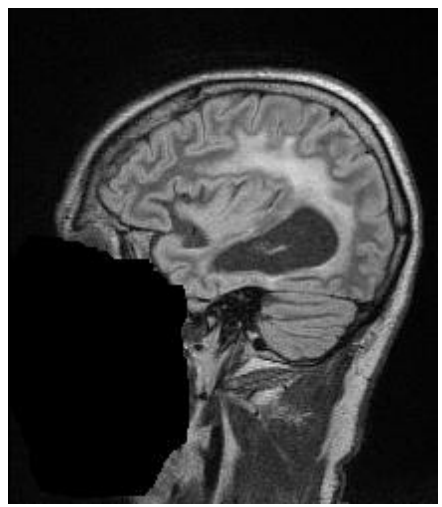

Fig. 2. A sample MR image of the brain in real size
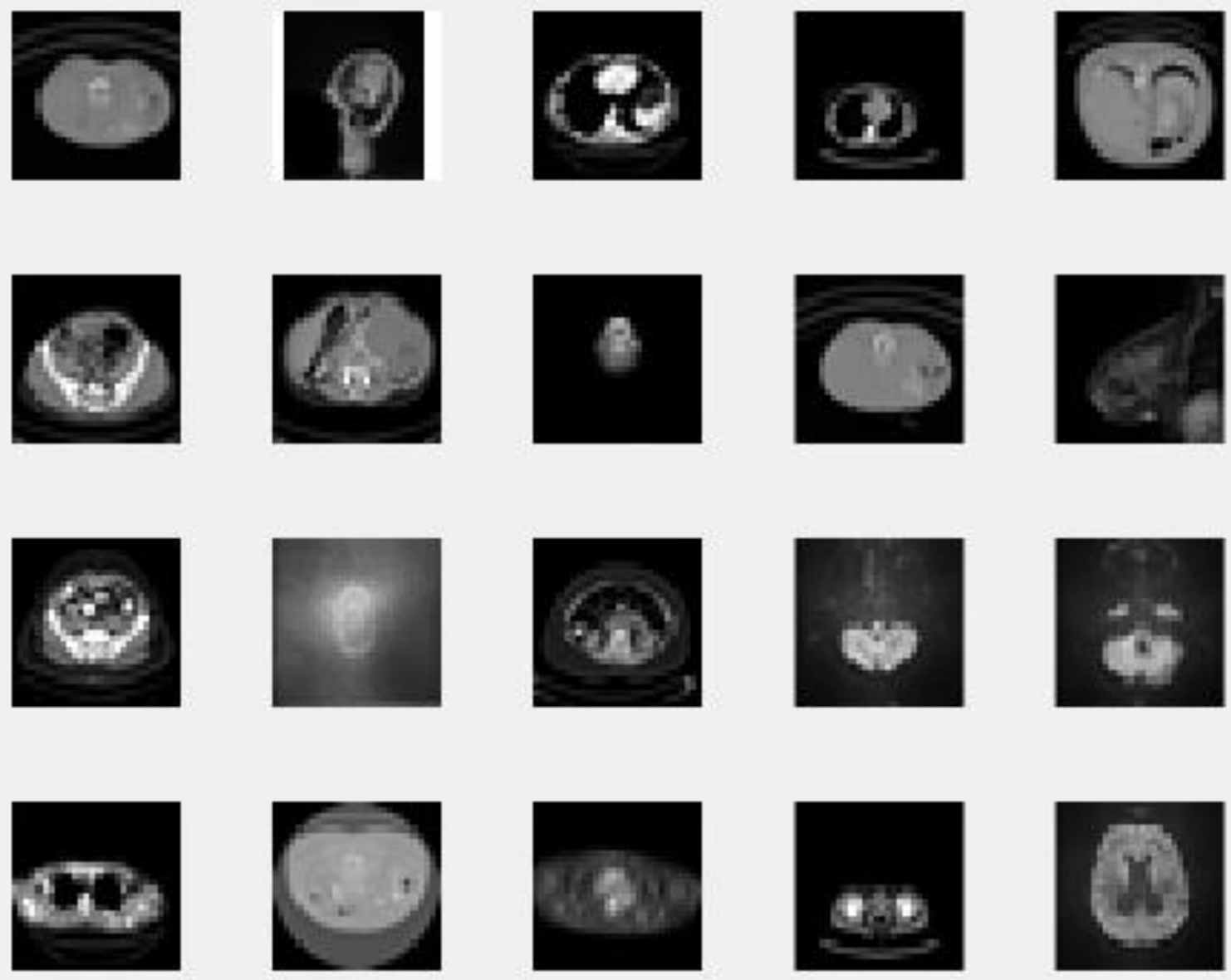

Fig. 3. Data set samples

\section{Methodology}

In the study, a deep convolutional neural network (DCNN) model, which is a kind of multi-layered perceptrons is used. Convolutional neural networks are an advanced deep learning approach based on artificial neural networks. In a convulsion neural network, there are generally the following layers: (i) the input layer is the layer at which the image data is recorded at the appropriate size and shape; (ii) the convolution layer is the layer from which a smaller pixel appearance is obtained using the appropriate filter; (iii) the ReLU layer is co-worked with the convolutional layer, the normalization function of the activation function; (iv) The Max Pooling layer is known as the pooling layer, and some sort of filtering is possible; The layer by which the maximum number of image codes are filtered out; (v) full connected and soft max layer, the layer where the full connection is made. In this layer, connections are made to all the outputs. After this layer, there is an output layer with a classification output [7 - 11]. Often these layers are repeated in the construction of DCNN architects. That is, 
two convolutional layers and two max pooling layers are used. In many studies, images can have multiple layers. If the images used are colored, they are images with 3 or more layers or sizes [11]. Two-dimensional black and white images were used in this study. The Deep Convolutional Neural Network model proposed in the study consists of 7 layers as shown in figure 4 . The DCNN model is simply designed as a single level. These 7 layers are input layer, convolution $2 \mathrm{~d}$ layer, relu layer, max pooling $2 \mathrm{~d}$ layer, full connected layer, softmax layer and classification layer which is the last layer. $75 \%$ of the data set was used for testing and the remaining $25 \%$ was used for testing [12-14].
In a DCNN model, the most important method to minimize the total error is to use the gradient descent algorithm. For the model proposed in the study, the mini-batch gradient descent (MBGD) algorithm is used. In the MBGD algorithm, a much smaller number of samples are used for each iteration of the total dataset. In the study, the Rectified Linear Unit (ReLU) activation function, shown in equation (1), is used. If the input value is less than zero, the value is zero [15].

$\operatorname{ReLU}(\mathrm{x})=\operatorname{Max}(0, \mathrm{x})$

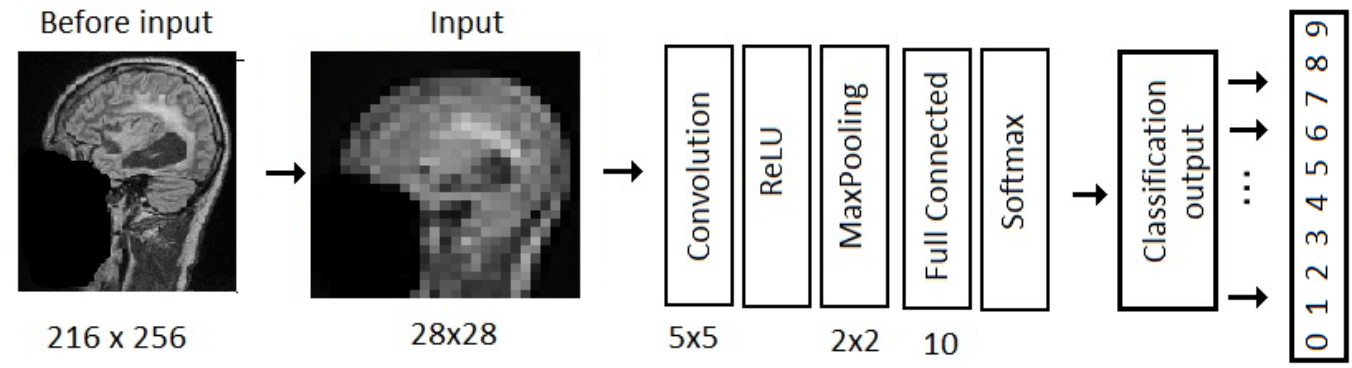

Fig. 4 Proposed DCNN architecture

In the convolution layer, a $5 \times 5$ filter was applied to the input image and the output was normalized by the ReLU activation function. After the ReLU layer, it was performed in 2 squares for the stride operation in the MaxPooling layer. The maximum epoch was selected as 15 for model training. The initial learning rate is 0.0001 as a starting point. In the next process, ten full connections are made for ten outputs.

\section{RESUlTS}

As a result of the test made, the accuracy rate for the classification was obtained as 0.9948 . Table 1 shows the training process of the model.

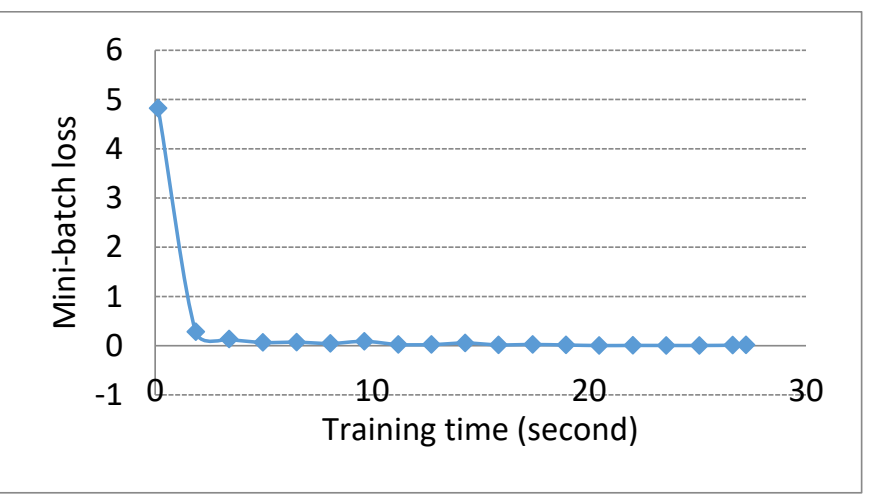

Fig.5 Traning process

As Table 1 reveals, in the 15 th epoch, a mini batc accuracy rate of $99.22 \%$ was achieved in 27.24 seconds, and a mini bathc loss was obtained in the 15 th epoch very close to zero at 0.00184 . Figure 5 shows the training process and loss rates, and figure 6 shows the DCNN model in terms of training and testing process, and finally, the point at which the DCNN model was reached.

TABLE I.

TRAINING PROCESS OF THE MODEL

\begin{tabular}{|c|c|c|c|c|}
\hline Epoch & Iteration & $\begin{array}{l}\text { Time Elapsed } \\
\text { Seconds) }\end{array}$ & $\begin{array}{l}\text { Mini-batch } \\
\text { Loss }\end{array}$ & $\begin{array}{l}\text { Mini - Batch } \\
\text { accuracy }\end{array}$ \\
\hline 1 & 1 & 0,14 & 4,8241 & 3,13 \\
\hline 1 & 50 & 1,87 & 0,2849 & 94,53 \\
\hline 2 & 100 & 3,41 & 0,1342 & 95,32 \\
\hline 3 & 150 & 4,97 & 0,0679 & 97,66 \\
\hline 4 & 200 & 6,52 & 0,0722 & 98,44 \\
\hline 5 & 250 & 8,08 & 0,0472 & 99,22 \\
\hline 6 & 300 & 9,64 & 0,0899 & 97,66 \\
\hline 7 & 350 & 11,21 & 0,0252 & 99,22 \\
\hline 7 & 400 & 12,74 & 0,0235 & 100 \\
\hline 8 & 450 & 14,29 & 0,0555 & 98,44 \\
\hline 9 & 500 & 15,83 & 0,0183 & 100 \\
\hline 10 & 550 & 17,4 & 0,0256 & 99,22 \\
\hline 11 & 600 & 18,94 & 0,0152 & 100 \\
\hline 12 & 650 & 20,47 & 0,0043 & 100 \\
\hline 13 & 700 & 22,02 & 0,0067 & 100 \\
\hline 13 & 750 & 23,56 & 0,0062 & 100 \\
\hline 14 & 800 & 25,09 & 0,0053 & 100 \\
\hline 15 & 850 & 26,62 & 0,0113 & 100 \\
\hline 15 & 870 & 27,24 & 0,0184 & 99,22 \\
\hline & & & & \\
\hline
\end{tabular}

The reason for ending Epoch 15 is that the error does not fall further from the 12th epoch but increases slightly. The lowest error level is reached in the 12th epoch $(0,0043)$. More importantly, the accuracy rate at the end of the first epoch begins with $94 \%$. 


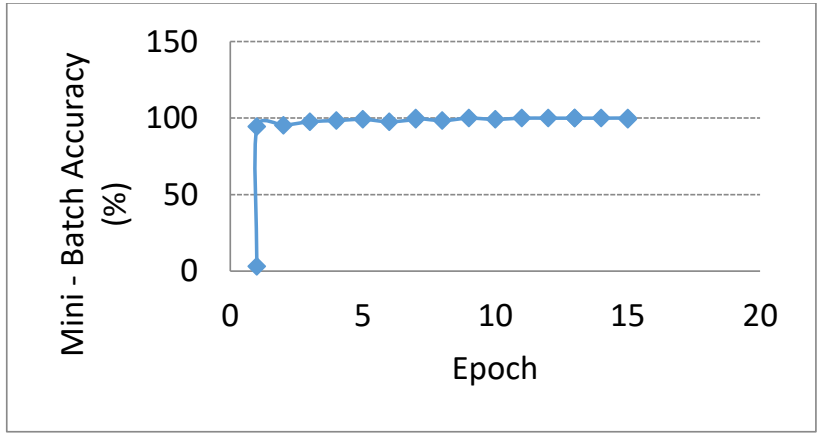

Fig. 6 Testing resut of the DCNN model

\section{CONCLUSIONS}

The training data used for the training of the proposed DCNN model in the study was reduced to a size of $28 \times 28$ pixels in the first stage. Although it is normally a very low pixel value, it is quite suitable for simple classification purposes only. On the other hand, it is very satisfactory that the accuracy rate obtained is 0.9948 . The study was performed in a MATLAB environment on a PC with GPU support. Therefore, the training process continued until the 27th minute. Despite the accuracy achieved for the classification or prediction of different types of cancer, the error rate is also very close to zero. This study has produced very successful results in light of future studies.

\section{REFERENCES}

[1]. U. Rajendra Acharya, Shu Lih Oh, Yuki Hagiwara, Jen Hong Tan, Hojjat Adeli, Deep convolutional neural network for the automated detection and diagnosis of seizure using EEG signals, Computers in Biology and Medicine, (2017) 1-9

[2]. M.E. Paoletti, J.M. Haut, J. Plaza, A. Plaza, A new deep convolutional neural network for fast hyperspectral image classification, ISPRS Journal of Photogrammetry and Remote Sensing , 31, May, 2017, 1-28

[3]. Pegah Khosravi, Ehsan Kazemi, Marcin Imielinski, Olivier Elemento, Iman Hajirasouliha, Deep Convolutional Neural Networks Enable Discrimination of Heterogeneous Digital Pathology Images, EbioMedicine, 2017, 1-12

[4]. Y. Zheng, Zhiguo Jiang, F. Xie, H. Zhang, Y. Ma, H. Shi, Yu Zhao Feature extraction from histopathological images based on nucleusguided convolutional neural network for breast lesion classification, Pattern Recognition 71 (2017) 14-25,

[5]. Vallières, M. et al. Radiomics strategies for risk assessment of tumour failure in head-and-neck cancer, Sci Rep, 7, 10117 (2017). doi: $10.1038 / \mathrm{s} 41598-017-10371-5$

[6]. http://www.cancerimagingarchive.net/, date of access: 10 Jan 2018,

[7]. M. Dais Ferreira, Débora Cristina Cor rêa, Luis Gustavo Nonato, Rodrigo Fernandes de Mello, Designing architectures of convolutional neural networks to solve practical problems, Expert Systems With Applications 94 (2018) 205-217

[8]. B. Krismono Triwijoyo, Widodo Budiharto, Edi Abdurachman, The Classification of Hypertensive Retinopathy using Convolutional Neural Network 2nd International Conference on Computer Science and Computational Intelligence 2017, ICCSCI 2017, 13-14 October 2017, Bali, Indonesia,

[9]. U. Rajendra Acharya, Shu Lih Oh, Yuki Hagiwara, Jen Hong Tan, Muhammad Adam, Arkadiusz Gertych, Ru San Tan, A deep convolutional neural network model to classify heartbeats, Computers in Biology and Medicine 89 (2017) 389-396

[10]. U.K. Lopes , J.F. Valiati, Pre-trained convolutional neural networks as feature extractors for tuberculosis detection, Computers in Biology and Medicine 89 (2017) 135-143.
[11]. M. Dyrmann, H. Karstoft, H. Skov Midtiby, Plant species classification using deep convolutional neural network, biosystems engineering, 151, (2016), $72-80$

[12]. S. Hussain, S. M. Anwar, M. Majid, Segmentation of glioma tumors in brain using deep convolutional neural network, Neurocomputing, (2017) $1-14$

[13]. H. Sharma, N. Zerbe, I. Klempert, O. Hellwich, P.Hufnagl, Deep convolutional neural networks for automatic classification ofgastric carcinoma using whole slide

[14]. S. Yu, SenJia, ChunyanXu, Convolutional neural networks for hyperspectral image classification, Neurocomputing, 219, (2017), 88-98

[15]. I. Goodfellow, J., Warde-farley, D., ve Courville. AMaxout Networks. Proceedings of the 30th International Conference on Machine Learning, Atlanta, Georgia, USA. JMLR: W\&CP.(2013). p. 28.

\section{BIOGRAPHIES}

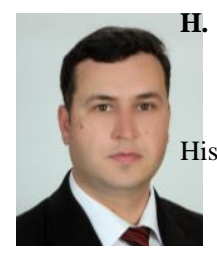

H. H. SELÇUK NOGAY received B.S degrees in Electrical Education from Kocaeli University, and M.S. and Ph.D degrees in Electrical Education from Marmara University respectially 2002, 2003 and 2008.

His research interests include Artificial Neural Network, Deep Learning and signal processing technique $\mathrm{He}$ has been working as a Professor in Vocational Scholl of Erciyes University in Kayseri, Turkey. 